\title{
The effects of resistance exercise training on arterial stiffness in metabolic syndrome
}

\author{
E. DeVallance ${ }^{1,2}$, S. Fournier ${ }^{1,2}$, K. Lemaster ${ }^{1,2}$, C. Moore ${ }^{1}$, S. Asano ${ }^{1,2}$, D. Bonner ${ }^{1}$, D. \\ Donley $^{1}$, I. M. Olfert ${ }^{1,2}$, and P.D. Chantler ${ }^{1,2}$ \\ P.D. Chantler: pchantler@hsc.wvu.edu \\ ${ }^{1}$ Division of Exercise Physiology, School of Medicine, West Virginia University, P.O. Box 9227, \\ Morgantown, WV 26506, USA \\ ${ }^{2}$ Center for Cardiovascular and Respiratory Sciences, School of Medicine, West Virginia \\ University, P.O. Box 9227, Morgantown, WV 26506, USA
}

\section{Abstract}

Purpose-Arterial stiffness is a strong independent risk factor for cardiovascular disease and is elevated in individuals with metabolic syndrome (MetS). Resistance training is a popular form of exercise that has beneficial effects on muscle mass, strength, balance and glucose control.

However, it is unknown whether resistance exercise training (RT) can lower arterial stiffness in patients with MetS. Thus, the aim of this study was to examine whether a progressive RT program would improve arterial stiffness in MetS.

Methods-A total of 57 subjects (28 healthy sedentary subjects; 29 MetS) were evaluated for arterial structure and function, including pulse wave velocity (cfPWV: arterial stiffness), before and after an 8-week period of RT or continuation of sedentary lifestyle.

Results-We found that 8 weeks of progressive RT increased skeletal muscle strength in both Con and MetS, but did not change arterial stiffness in either MetS (cfPWV; Pre $7.9 \pm 0.4 \mathrm{~m} / \mathrm{s}$ vs. Post $7.7 \pm 0.4 \mathrm{~m} / \mathrm{s}$ ) or healthy controls (cfPWV; Pre $6.9 \pm 0.3 \mathrm{~m} / \mathrm{s}$ vs. Post $7.0 \pm 0.3 \mathrm{~m} / \mathrm{s}$ ).

However, when cfPWV is considered as a continuous variable, high baseline measures of cfPWV tended to show a decrease in cfPWV following RT.

Conclusion-Eight weeks of progressive RT did not decrease the group mean values of arterial stiffness in individuals with MetS or healthy controls.

\section{Keywords}

Arterial stiffness; Exercise; Central hemodynamics; Metabolic syndrome

Correspondence to: P.D. Chantler, pchant ler@hsc . wvu . edu.

Compliance with ethical standards

Conflict of interest The authors declare no competing financial interests. 


\section{Introduction}

Arterial stiffness (AS) is a strong independent predictor of cardiovascular (CV) mortality (Lakatta and Levy 2003). Increased AS is a common feature of metabolic syndrome (MetS) (Donley et al. 2014; Scuteri et al. 2004; Vyssoulis et al. 2010) and is one of the driving forces behind the increased CV risk in patients with MetS. The mechanisms through which MetS increases AS are likely to be multi-factorial. Reduced endothelial function via a reduction in nitric oxide bioavailability, combined with elevated central pressures, increases vascular tone, shifting forces onto the more stiffer collagen fibers (Lakatta and Levy 2003; Scuteri et al. 2004; Stepp 2006; Wilkinson et al. 2004). Hypertension increases collagen deposition and decreases elastin (Kato et al. 1991; Levy et al. 1993; Rattazzi et al. 2012). Further, a hyperglycemic state likely results in an increased accumulation of advanced glycation end products and the non-enzymatic glycation of matrix proteins, increasing AS in MetS (Emre et al. 2009). A stiffer arterial system can increase central systolic blood pressure (cSBP) leading to increasing cardiac work and decreasing diastolic blood pressure, which in turn can decrease coronary blood flow (Lakatta and Levy 2003). Therapeutic interventions aimed at lowering AS in individuals with MetS are increasingly being seen as important to slow or reverse the progression of CV disease.

Resistance training (RT) is a popular form of exercise that has been shown to exert beneficial effects on muscle mass (improved insulin sensitivity and weight loss), while reducing the risk of sarcopenia, osteoporosis, and frailty (Ibanez et al. 2005). RT is also recognized and recommended by the American Heart Association as a beneficial therapy to help improve the CV health of diseased populations (Williams et al. 2007). Although we have previously shown that aerobic exercise training for 8 weeks reduces AS in subjects with MetS (Donley et al. 2014; Fournier et al. 2015), what effect RT has on AS in MetS is unknown. Previous studies in healthy young and middle-aged subjects suggest that AS increases after RT (Cortez-Cooper et al. 2005; Miyachi et al. 2003, 2004), while other studies showed no change or a decrease with RT (Casey et al. 2007; Heffernan et al. 2009; Rakobowchuk et al. 2005). Furthermore, studies conducted using healthy young or middle-aged obese individuals with fairly healthy measures of AS, have shown no alterations in central AS or augmentation index (an indirect measure of AS) (Croymans et al. 2014; Ho et al. 2012). These differences likely arise from the variations in training programs with high intensity or failure training eliciting increases in AS and beneficial or no change coming from progressive moderate programs. Importantly, it remains unknown in individuals with MetS (but still symptoms for overt CV disease or diabetes absent) whether RT would be beneficial or have an adverse effect on AS. Therefore, the purpose of this study was to examine the effects of RT on AS in individuals with MetS (without overt CV disease or diabetes). Further, we wanted to establish if the RT response to AS differed in those with MetS compared to healthy middle-aged subjects. We hypothesized based on data from similar training programs that a progressive 8-week RT program for those with MetS would not alter AS, but significantly improve skeletal muscular strength and aerobic capacity. We also hypothesized that the RT response would be similar between MetS and healthy controls. 


\section{Methods}

\section{Study population}

Initially, 30 healthy controls and 30 subjects with MetS consented to participate in the study. Individuals were randomly assigned using opaque envelopes into either an RT intervention group or a non-exercised control (Con) group for 8 weeks. However, three subjects (two healthy, one MetS) dropped out due to personal reasons or injuries sustained outside of the research protocol. In total, 28 healthy controls (46 \pm 11 year; $72 \%$ female) and 29 MetS (51 \pm 12 year; $70 \%$ female) subjects completed the 8 -week study with $\geq 90 \%$ adherence (completed at least 22 of 24 workouts) to the exercise protocol (Fig. 1). The healthy and MetS Con groups maintained their normal lifestyle (time control) for 8 weeks. We selected 8 weeks of RT, because we have previously shown that 8 weeks of aerobic exercise training is beneficial in lowering AS by approximately $10 \%$ (Donley et al. 2014), which may reflect a lowering of CV disease events by approximately $14 \%$ (Vlachopoulos et al. 2010).

The healthy and MetS subjects were free from overt CV disease and diabetes, as determined by a medical history, physical examination, and a normal resting and exercise electrocardiogram. Furthermore, none of the participants were current or former smokers. MetS was defined according to the updated National Cholesterol Education Program, Adult Treatment Panel III (Huang 2009) composed of three out of the following five components:

1. obesity (waist: $\mathrm{men}>102 \mathrm{~cm}$, women $>88 \mathrm{~cm}$ ).

2. low high-density lipoprotein cholesterol (men $<1.03 \mathrm{mmol} / \mathrm{L}$; women $<1.29$ $\mathrm{mmol} / \mathrm{L})$.

3. hypertriglyceridemia (>1.7 mmol/L).

4. elevated glucose $(>5.6$ and $<7.0 \mathrm{mmol} / \mathrm{L})$.

5. elevated blood pressure (BP) (130/85 $\mathrm{mmHg}$ or use of hypertensive medications).

Additionally, exclusion criteria included type 2 diabetes mellitus (hemoglobin A1c >6.5\%, use of diabetic medications, or doctor's diagnosis), pulmonary disease, angina, atrial fibrillation, aortic stenosis, anemia, myocardial infarction, stroke, or coronary revascularization. Subjects who participated in regular exercise, defined as >30 min, 3 times/ week were also excluded.

This study was approved by the West Virginia University Institutional Review Board, and all procedures were in accordance with the ethical standards of the 1964 Helsinki Declaration and its later amendments. All subjects provided informed written consent prior to participation.

\section{Study design}

Assessments were performed between 7:00 and 10:00 AM, in a quiet, temperaturecontrolled room after a 12-h fast and abstinence from alcohol, caffeine, and vitamins. Blood pressure and cholesterol medications were withheld $24 \mathrm{~h}$ before assessments. On completion of the anthropometric assessments and after a minimum of $15 \mathrm{~min}$ of quiet supine rest, subjects underwent supine measures of arterial structure and function followed by a blood 
draw. All pre- and post-measurements were performed at the same time of day for each subject and at least $48 \mathrm{~h}$ after the last exercise session to avoid the immediate effects of a single bout of exercise.

\section{Body anthropometry}

Height and weight, along with waist and hip circumference, were measured using standard laboratory procedures. Fat distribution was assessed by measuring the waist circumference at the site of the smallest circumference between the rib cage and the iliac crest, with the subjects in a standing position. Body composition was calculated from body volume by the BodPod (Life Measurement, Concord, CA). Body mass index was calculated as: weight $(\mathrm{kg}) /$ height $^{2}(\mathrm{~m})$.

\section{Metabolic indices}

Venous blood sampling was obtained in the morning after a 12-h overnight fast. Posttraining venous samples were collected at least $48 \mathrm{~h}$ after the last exercise session. Plasma obtained from blood sampling was analyzed at West Virginia University Hospital's central laboratory in Morgan-town, West Virginia. Total cholesterol, HDL, triglycerides, and glucose were determined in plasma (lithium heparin, Becton Dickenson Plasma Separator Tubes) using Beck-man-Coulter (CA, USA) DxC automated chemistry analyzers. Total cholesterol was measured using a cholesterol esterase/cholesterol oxidase/peroxidase-driven, timed-end-point method (coefficients of variation $<7 \%$ ). HDL cholesterol was measured using a cholesterol esterase/cholesterol oxidase/peroxidase-driven, timed-endpoint assay with automated initial homogeneous solubilization step (coefficients of variation $<7 \%$ ). Triglycerides were measured using a lipase/glycerol kinase/glycerophosphate oxidase/ horseradish peroxidase-driven, timed-endpoint method (coefficients of variation 5-7 \%). Glucose was measured electrochemically using a glucose oxidase/catalase/molybdate-driven oxygen rate method (coefficients of variation $<5 \%$ ). Glycated hemoglobin (A1c fraction) was measured in whole blood ( $\mathrm{K}_{2}$-EDTA, Becton Dickenson) using a BioRad (Hercules, CA, USA) Variant II Turbo High Performance Liquid Chromatography (HPLC) system (coefficients of variation $<2 \%$ ). Insulin was measured in serum (untreated/ red-top tube, Becton Dickenson) on an Immulite 2000 immunochemistry system (Siemens, USA; coefficients of variation $<10 \%$ ).

\section{Arterial structure and function}

Methods for arterial geometry and function, exercise testing, and metabolic indices are provided in brief below. A detailed description of these methods can be found in our laboratory's previously published papers (DeVallance et al. 2015; Donley et al. 2014). A single investigator blinded to group allocations performed measurements. The interclass correlation coefficients (ICC) and coefficient of variations for our laboratory have been previously reported (DeVallance et al. 2015) with minimum ICC $=0.94$ and maximum variation of $6 \%$ for any measurement.

Arterial geometry-Ultrasound (GE Vivid i, Toronto, Canada) 2-D images of the right common carotid artery (CCA) were obtained containing the carotid bifurcation and $>2 \mathrm{~cm}$ of the proximal CCA. Images were used to measure lumen diameter and intima-medial 
thickness (IMT). At rest, the ICC and the coefficient of variation, collected on two separate days, for IMT was: coefficient of variation $=3 \%$, and ICC $=0.96$.

Arterial function-Brachial systolic (bSBP) and diastolic (bDBP) blood pressure was measured with an automated, oscillometric, sphygmomanometer (Critikon Dinamap Compact BP monitor, GE Medical, Tampa, Fla, USA) and pulse pressure (bPP) was calculated from bSBP-bDBP. Pulse wave analysis was performed noninvasively on the radial artery (SphygmoCor system, AtCor Medical, Sydney, Australia). The Sphygmo-Cor system synthesizes a central (ascending aortic) pressure waveform from the radial pressure waveform that does not differ from that of an intra-arterially recorded wave (Chen et al. 1996) using a validated generalized transfer function (Chen et al. 1997) that has good reproducibility under major hemodynamic changes (Sharman et al. 2006). These waveforms were calibrated against brachial mean arterial and diastolic pressure to estimate aortic pressures. From the central pressure wave, central systolic (cSBP), diastolic (cDBP), pulse pressure ( $\mathrm{cPP})$, augmented pressure (AP), and the pressure at the forward wave peak, and central augmentation index (AGI) were determined. All measurements were made in triplicate and the mean values used for subsequent analysis. Pressure waveforms with an operator index of $>75 \%$ were determined suitable for examination.

Carotid to femoral pulse wave velocity (cfPWV; central arterial stiffness) was measured by applanation tonometry (AtCor Medical, Sydney, Australia). ECG-gated waveforms were sequentially recorded. The aortic distance was calculated as the difference in the distances from the carotid to the suprasternal notch and from the suprasternal notch to the femoral artery or radial artery. The time delay was calculated using a foot-of-the-wave method. Of note, the coefficient of variation and ICC for cfPWV from our laboratory was $3 \%$ and 0.98 , respectively.

\section{Aerobic capacity testing}

Subjects exercised to exhaustion on a modified Monarch bike to obtain subjects' maximum aerobic capacity. The pedal speed was set at $50 \mathrm{rpm}$, and workloads increased by $25 \mathrm{~W}$ every 3 min until exhaustion. Peak oxygen consumed $\left(\mathrm{VO}_{2 \text { peak }}\right)$ was measured from breath-bybreath gas analysis (ParvoMedics, Sandy, UT) and verified by subjects achieving a peak HR $>85 \%$ predicted max, an RER $>1.0$, and a BORG scale $>18$. Subjects had ECG, and heart rate was continuously monitored over the duration of the exercise test, while BP was measured at the end of each exercise stage and prior to maximal effort by experienced exercise physiologists.

\section{Resistance training intervention}

MetS and healthy individuals were randomly selected to participate in an RT program for 3 days/week and consisted of six exercises (leg press, chest press, lat pull down, leg curl, shoulder press, and leg extension) on body master weight machines. The exercise intensity was based on individual's results from a one-repetition maximum (1RM). Subjects completed 1RM testing in the week prior to beginning the RT intervention. Briefly, subjects were familiarized to each machine with three warm-up sets of minimal resistance, followed by sets of five and three repetitions at increasing resistance. Testing finished with one 
repetition at increasing resistance until the resistance could not be lifted with proper form; this was repeated for all six lifts. RT intensities were increased progressively in 2-week increments: weeks $1-2,60 \%$ of max; weeks $3-4,70 \%$ of max; weeks $5-6,80 \%$ of max; and weeks $7-8,85 \%$ of max. Subjects were encouraged to complete three sets of 8-12 repetitions to fatigue, but not failure. The load and repetition range comply with those for hypertrophic training set by the American College of Sports Medicine (ACSM) and the National Strength and Conditioning Association and also comply with ACSM's stance on RT for health in an adult population (Baechle et al. 2008; Kraemer et al. 2002). All training was performed in the Human Performance Lab at West Virginia University and adherence was monitored through exercise logs filled out by staff.

\section{Statistical analysis}

Normality of data distribution was evaluated by the Kolmogorov-Smirnov test. Categorical variables were compared by the $\chi^{2}$ test. Continuous variables were log transformed as necessary, and pre-exercise variables were compared between groups with a one-way ANOVA with Tukey's post hoc test. Two-way repeated-measures ANOVA was used to evaluate the effects of exercise training with a time-by-group interaction to determine whether differences existed between group (Mets vs. healthy controls) and condition (RT vs. non-RT). The primary outcome measure was cfPWV and the secondary outcome measure was SBP. The RT group data were pooled and bivariate correlations were run on baseline measurements to the change in cfPWV following the 8 weeks of RT. Further, a backward elimination model was used to determine which of the correlated measurements were predictive of the cfPWV response. All analyses were performed with the statistical package SPSS version 22 (SPSS, Chicago, IL). All values represent mean \pm SEM, unless otherwise stated. $P \leq 0.05$ was defined as significant. Using G-power (version 3.19.2, Germany), sample size calculation for our primary outcome measure cfPWV requires at least 12 subjects to detect a clinically significant effect of hypertrophic RT on AS, such as a difference in cfPWV of $1.0 \pm 1.0(\mathrm{SD}) \mathrm{m} / \mathrm{s}$ (Vlachopoulos et al. 2010) assuming a power of $85 \%$ and a error probability of 0.05 .

\section{Results}

\section{Anthropometric/training}

As expected based on study design, MetS groups had significantly elevated levels of blood lipids, abdominal/overall obesity, and blood pressure at baseline compared to the control groups (Table 1). Importantly, there were no significant group differences for sex or age (Table 1). There were also no differences in lipid profile or anthropometric measures between the two respective MetS groups, or between the two Con groups (Table 1, Table 2) with two exceptions: baseline triglycerides were higher in the Con compared to the healthy Con RT group and hemoglobin A1c was high in MetS compared to MetS RT.

After the exercise intervention, the MetS RT group showed a non-significant $11 \%$ decrease $(p=0.07)$ in body fat (Table 2). Also, the MetS RT group had a $12 \%$ increase $(p \leq 0.05)$ in $\mathrm{VO}_{2 \text { peak }}$, while no change was found with the Con RT, or among the sedentary (nonexercising) Con or MetS groups (Fig. 2). Muscular strength (measured via 1RM) was 
increased in all RT subjects for all six lifts; however, MetS RT showed significantly greater improvements in leg press, bench press, leg curl, and shoulder press (Table 2).

\section{Arterial function and structure}

MetS subjects exhibited higher baseline cfPWV ( 16\% faster central AS, $p<0.05)$ compared to healthy subjects, where the combined average for both MetS groups was 7.9 $\pm 0.4 \mathrm{~m} / \mathrm{s}$ and the combined average for the healthy subjects was $6.8 \pm 0.3 \mathrm{~m} / \mathrm{s}$. Further, bSBP, cSBP, bPP, and cPP were higher in the MetS groups compared to the healthy subjects (Table 3). Following RT, central AS (cfPWV), whose baseline values in the MetS RT were greater than Con RT, also remained unchanged with RT (Fig. 3). As shown in Table 3, RT resulted in no changes in any measure of arterial function, namely AP, and AGI.

The combined baseline average for CCA and IMT was significantly greater in MetS (by 5 and $12 \%$, respectively) compared to healthy subjects (Table 3 ). There were no significant changes in CCA or IMT with the RT program in either group, suggesting that 8 weeks of RT produced no changes in arterial structure.

\section{Predisposition to PWV improvement}

Since we found no significant changes in arterial structure or function following RT in either MetS or Con groups, we pooled together the RT data from both groups (creating a more comprehensive data set) to evaluate the potential contribution for baseline anthropometric and/or arterial structure/function to change cfPWV following 8 weeks of RT. We found a significant inverse relationship between delta (visit 2-visit 1) cfPWV and baseline measurements of cfPWV $(r=-0.67), \operatorname{cSBP}(r=-0.52)$, bSBP $(r=-0.48)$, and IMT $(r=$ $-0.45)$. Next, we included age, sex, and MetS with cfPWV, bSBP, cSBP, or IMT in a backward-linear elimination regression model to determine the predictability of these measures of decreasing cfPWV with RT. Here, we found that baseline cfPWV was the best predictor of changes in cfPWV $(r=-0.67, p<0.01)$. However, we also ran the backward elimination without cfPWV to determine if any indirect measure was predictive of the change and found both central $(r=-0.52, p<0.01)$ and peripheral $(r=-0.48, p<0.01)$ SBP to be predictive.

\section{Discussion}

The principal finding in this study is that progressive RT for 8 weeks did not change AS in either the MetS or healthy control groups. However, significant improvement was obtained in muscle strength in both MetS and Con RT groups. Additionally, the aerobic capacity increased in MetS RT. Existing literature reporting on the effects of RT on AS have produced a wide range of results, suggesting increased (Cortez-Cooper et al. 2005; Miyachi et al. 2003, 2004), no change (Casey et al. 2007; Cortez-Cooper et al. 2005; Croymans et al. 2014; Rakobowchuk et al. 2005), or decreased (Beck et al. 2013) effect on AS in either healthy and obese young or middle-aged individuals. Until now, the effects of RT on AS in MetS have been unknown. Given that individuals with MetS are known to have elevated baseline AS leaving them at greater risk of CV events (Donley et al. 2014; Fournier et al. 2014, 2015), any intervention that might increase (i.e., worsen) AS would be detrimental to 
overall CV health. In a previous study, we have shown that aerobic exercise training (for 8 weeks) decreased cfPWV in MetS (Donley et al. 2014). In contrast, we found that RT did not decrease cfPWV in MetS. It is, however, equally important to note that we found no detrimental effects of RT on AS in either our MetS or healthy control subjects.

Previous studies have established that an increase in AS after RT typically emanated from young healthy men following high-intensity RT (Cortez-Cooper et al. 2005; Miyachi et al. 2003, 2004), with the exception of Casey et al., who found no change in cfPWV in young men following high-intensity RT (Casey et al. 2007). It has been suggested that the type of RT performed is likely to be important when considering its effects on AS. For example, in the study by Miyachi et al., where RT increased arterial dysfunction (AGI and carotid compliance), the RT protocol was a hypertrophic set $\times$ repetition scheme with intensity set at $80 \%$ for the entire duration of the study (Miyachi et al. 2004). Further, every third set was completed to failure, which is a high-intensity stimulus. Similarly, Cortez et al. obtained an increase in AS after RT, which incorporated high-intensity failure sets, suggesting that highintensity or failure RT may be the stimulus for increased AS (Cortez-Cooper et al. 2005), whereas the study by Casey et al. incorporated a progressive RT program and found no effect on AS (Casey et al. 2007). Although the current study utilized multiple similar muscle groups, the total volume of training, as reflected by the number of total sets $\times$ total repetitions $\times$ resistance, was substantially less than that reported in previous studies (CortezCooper et al. 2005). Further, the current study incorporated a progressive RT program that began at a moderate intensity. Of note, a recent meta-analysis found that this may not be exclusive to RT, suggesting that even higher-intensity, weight-bearing aerobic training can have adverse effects (Montero et al. 2014). It was suggested that the intense muscle contractions needed to complete weight-bearing aerobic training may increase intramuscular pressure, leading to high wall stress in the arteries, resulting in wall remodeling and therefore increased AS (Montero et al. 2014). However, in our study, we implemented a progressive, moderate RT program. Our results in MetS are similar to that identified (i.e., no alterations in cfPWV or AGI) in obese individuals (a single component of MetS) (Croymans et al. 2014; Ho et al. 2012). Therefore, it seems likely that differing styles and intensities of RT interventions may have important consequences on the health and function of the arterial system. This needs to be more carefully evaluated in future studies. Taken together, we believe these data suggest that there is little concern that RT might be detrimental to AS in the context of MetS.

Although we did not find a group change in AS after RT, the backward elimination models suggested that those individuals with a higher baseline cfPWV (using our pooled data) was a predictor of individuals decreasing AS post-RT (Table 4). It is well documented, especially in the Heritage Family study (Bouchard et al. 1999), that not everyone is a 'responder' to the exercise training stimulus. A number of articles, which include aerobic training, have looked at demographic and anthropometric predictors of training outcomes (Bouchard 2012; Bouchard and Rankinen 2001; Skinner et al. 2001; Thomas et al. 1985). A recent study identified that RT did not alter AGI in middle-aged obese individuals (Ho et al. 2012). However, once the obese subjects were separated into 'responders' and 'non-responders' based on a 1 unit decrease in AGI, it was found that their form of RT decreased AGI similar to that seen in the aerobic and combination of RT and aerobic exercise groups. Taken 
together with our data, this suggests that individuals with the stiffest arteries gained a beneficial lowering of their AS after 8 weeks of progressive RT. However, this correlation may not be unique to RT, as individuals with the highest PWV may benefit from any form of physical activity, because they have the greatest room for improvement. Conversely, does this mean that healthy individuals with 'normal' age-associated AS values will not benefit from regular exercise training? We have previously shown that aerobic exercise training in healthy middle-aged individuals significantly lowered AS (Donley et al. 2014). Similarly, other groups have also found that exercise can lower AS in healthy individuals (Currie et al. 2009; Tanaka et al. 2000). The mechanisms behind these improvements likely reflect enhanced endothelial function (a key component of AS), along with changes in the intrinsic properties of the vessel wall (smooth muscle content, collagen and elastin properties). Such adaptations in elastin and collagen have been confirmed in animal studies after exercise interventions (Fleenor et al. 2010; Kingwell et al. 1997; Matsuda et al. 1993; Seals 2014). Another mechanism, which may influence vascular adaptations to RT, is the increased pressures caused by Valsalva-like maneuvers. It is unknown how the specific forces exerted by this maneuver control vascular adaptation to training. As depicted in Fig. 4, those with the most compliant aortas suffered the greatest increase in cfPWV, which may highlight a differing effect of Valsalva. This suggests that adaptations to high aortic pressures in compliant vessels could become stiffer, while vessels that are already stiff are unaffected. To what extent this stimulus causes vascular adaption to RT is unknown and warrants future investigation. Although baseline cfPWV was the strongest predictor of a decrease in PWV via RT, we also identified that baseline bSBP (an important component of AS) was also a good predictor of the drop in cfPWV post-RT. Further, this relationship remained after we removed five individuals on hypertensive medications (data not shown), given that the halflife of hypertensive medications is approximately 2 days. This suggests that in the absence of cfPWV measurements, an individual's peripheral SBP could be used to determine if they are likely to gain $\mathrm{CV}$ improvements from progressive RT.

While the benefits of our progressive RT program were limited to gains in skeletal muscle strength, it should be noted that low muscle strength and mass are often associated with the development of MetS (Atlantis et al. 2009). Muscle mass and strength are also found to be highly predictive of a subject's insulin resistance (Atlantis et al. 2009). As such, RT may also be beneficial in slowing the progression of MetS patients to overt CV disease. Indeed, we saw a clinically relevant trend (albeit, not statistically significant) for decrease in triglycerides in our MetS RT group. We also demonstrated greater increases in muscle strength in MetS compared to Con RT. The early adaptations to resistance training are mainly neurological and may have a greater effect on the slightly $(p=0.1)$ higher lean mass noted in MetS RT ( $54 \pm 3 \mathrm{~kg}$ ) compared to Con RT ( $49 \pm 2 \mathrm{~kg})$, and the increase in type II muscle fibers associated with MetS (Miller et al. 2015; Stuart et al. 2013). However, the mechanisms of strength gains in MetS were beyond the scope of this study, so we also cannot rule out other factors. RT-mediated increases in muscle mass are also known to elicit a beneficial shift in body composition (Chilibeck et al. 1998; Hunter et al. 2004; Yarasheski et al. 1999), and here again we observed a trend for decrease in body fat \% in MetS with RT. While these changes were not statistically significant, and not enough to change total metabolic risk assessment, continued participation in RT would be expected to produce 
significant CV benefits (Brown et al. 2001; Cullen 2000; Jacobson et al. 2007). This could mean that longer interventional durations of hypertrophic RT are needed in a MetS population to produce those physiological changes and elicit reduction of $\mathrm{CV}$ risk factors that could alter the MetS classification.

\section{Limitations}

There are several limitations. First, the sample size for our training and non-training group was modest $(n=13-16)$ and a subsequent power analysis revealed that we were underpowered for cfPWV (statistical power $=0.10 \%$ ). As such, we cannot exclude the possibility of a type II statistical error (i.e., that we have falsely accepted the null hypothesis). However, we have previously shown that a similar sample size was sufficient to identify a significant decrease in cfPWV after aerobic training (Donley et al. 2014). Further, the $0.1 \mathrm{~m} / \mathrm{s}$ non-significant decrease in cfPWV in the Mets RT group would not be considered clinically relevant even if statistically significant.

A second limitation of the study involves medications. While none of the healthy subjects were on any medications, MetS patients were on hypertensive and/or cholesterol medications, which may have influenced the exercise responses. Although all subjects withheld their blood pressure medications $24 \mathrm{~h}$ prior to the physiological measurements, we cannot rule out that the chronic effects of these medications may have impacted the CV parameters. Further, we explored the relationship between baseline PWV and the change in PWV pre- to post-RT removing the five subjects on hypertensive medications, and found that the relationship was not significantly changed (data not shown). Importantly, the MetS population examined in our study is a reflection of individuals with multiple risk factors undergoing medical treatment for those risk factors.

Third, our sample population consisted of women who were pre- $(33 \%)$ and postmenopausal $(67 \%)$, of which none of the post-menopausal women were on hormonal replacement therapy. No differences or trends were identified in the change in cfPWV following RT in either pre-or post-menopausal groups in either MetS or healthy RT groups (data not shown). As expected, when we examined by menopausal status, post-menopausal women had higher cfPWV values compared to pre-menopausal women (data not shown). Furthermore, previous work has suggested that sex differences are an important variable for RT outcome (Collier et al. 2011). Although our backward regression models showed no effect of sex on changes in cfPWV following RT, a limitation of this study is that we were not powered to examine sex differences. Thus, future research is required to examine the sex-specific, along with the menopausal effects of RT (with various forms of RT) on AS.

Fourth, subjects were asked to maintain their normal lifestyle that included diet and physical activity (for the no RT groups), which were checked via weekly phone calls or during the subjects' visit to the human performance laboratory. However, we did not utilize food or physical activity logs and as such we recognize that as a limitation in our study design. We did, however, have exercise logs for the RT groups. 
Finally, the short duration of exercise training ( 8 weeks) may have been insufficient to alter AS. We chose to examine 8 weeks of exercise training as we have previously shown beneficial effects of 8 weeks of aerobic training in MetS on AS. Based on the varying AS responses to RT found in the existing literature, we also cannot exclude the possibility that longer duration RT may not also ultimately alter AS, in either a positive (i.e., decreased PWV) or negative (i.e., increased PWV) manner relative to CV health. Thus, longer exercise training programs are important to fully understand the role that exercise training has on improving CV structure/function in patients with MetS.

In summary, in our population of MetS, 8 weeks of progressive RT produced significant changes in muscle strength, but did not significantly alter the indices of arterial structure and function related to the assessment of arterial stiffness. Our backward regression modeling found that those with the stiffest vessels had the largest reduction in PWV following the 8 weeks of RT, suggesting that significant changes in AS in association with RT (or perhaps any form of) exercise may only be fully realized by individuals with arterial dysfunction.

\section{Acknowledgments}

We thank Beth Nardella for proofing the manuscript. This study was supported in part by the American Heart Association Grant 11CRP7370056 (P. D. Chantler), National Heart, Lung, and Blood Institute Grant T32HL-090610 (S. B. Fournier), and National Institute of General Medical Sciences of the National Institutes of Health under Award U54-GM-104942 and 1P20 GM109098 (P. D. Chantler). STEM Mountains of Excellence Fellowship (E. DeVallance). The content is solely the responsibility of the authors and does not necessarily represent the official views of the National Institutes of Health.

\section{Abbreviations}

1RM One repetition maximum

AGI

Augmentation index

ANOVA

Analysis of variance

AP

Augmented pressure

AS

Arterial stiffness

bDBP

Brachial diastolic blood pressure

bPP

Brachial pulse pressure

bSBP

Brachial systolic blood pressure

CCA Common carotid artery

cfPWV Carotid to femoral pulse wave velocity

Con Control

cSBP Central systolic blood pressure

CV Cardiovascular

DBP Diastolic blood pressure

HDL High-density lipoprotein 


$\begin{array}{ll}\text { ICC } & \text { Interclass correlation coefficients } \\ \text { IMT } & \text { Intima-medial thickness } \\ \text { MetS } & \text { Metabolic syndrome } \\ \text { RT } & \text { Resistance training } \\ \text { SEM } & \text { Standard error of the mean } \\ \text { VO2peak } & \text { Peak oxygen consumption }\end{array}$

\section{References}

Atlantis E, Martin SA, Haren MT, Taylor AW, Wittert GA. Members of the Florey Adelaide Male Ageing S. Inverse associations between muscle mass, strength, and the metabolic syndrome. Metab Clin Exp. 2009; 58:1013-1022. [PubMed: 19394973]

Baechle, TR.; Earle, RW. Essentials of strength training and conditioning. 3rd. Champaign: Human Kinetics; 2008. National Strength \& Conditioning Association (US).

Beck DT, Martin JS, Casey DP, Braith RW. Exercise training reduces peripheral arterial stiffness and myocardial oxygen demand in young prehypertensive subjects. Am J Hypertens. 2013; 26:10931102. [PubMed: 23736111]

Bouchard C. Genomic predictors of trainability. Exp Physiol. 2012; 97:347-352. [PubMed: 21967902]

Bouchard C, Rankinen T. Individual differences in response to regular physical activity. Med Sci Sports Exerc. 2001; 33:S446-S451. discussion S452-S443. [PubMed: 11427769]

Bouchard C, et al. Familial aggregation of $\mathrm{VO}(2 \mathrm{max})$ response to exercise training: results from the HERITAGE Family Study. J Appl Physiol. 1999; 87:1003-1008. [PubMed: 10484570]

Brown BG, et al. Simvastatin and niacin, antioxidant vitamins, or the combination for the prevention of coronary disease. N Engl J Med. 2001; 345:1583-1592. [PubMed: 11757504]

Casey DP, Beck DT, Braith RW. Progressive resistance training without volume increases does not alter arterial stiffness and aortic wave reflection. Exp Biol Med. 2007; 232:1228-1235.

Chen $\mathrm{CH}$, et al. Validation of carotid artery tonometry as a means of estimating augmentation index of ascending aortic pressure. Hypertension. 1996; 27:168-175. [PubMed: 8567037]

Chen CH, Nevo E, Fetics B, Pak PH, Yin FCP, Maughan WL, Kass DA. Estimation of central aortic pressure waveform by mathematical transformation of radial tonometry pressure: validation of generalized transfer function. Circulation. 1997; 95:1827-1836. [PubMed: 9107170]

Chilibeck PD, Calder AW, Sale DG, Webber CE. A comparison of strength and muscle mass increases during resistance training in young women. Eur J Appl Physiol Occup Physiol. 1998; 77:170-175. [PubMed: 9459538]

Collier SR, Frechette V, Sandberg K, Schafer P, Ji H, Smulyan H, Fernhall B. Sex differences in resting hemodynamics and arterial stiffness following 4 weeks of resistance versus aerobic exercise training in individuals with pre-hypertension to stage 1 hypertension. Biol Sex Differ. 2011; 2:9. [PubMed: 21867499]

Cortez-Cooper MY, DeVan AE, Anton MM, Farrar RP, Beckwith KA, Todd JS, Tanaka H. Effects of high intensity resistance training on arterial stiffness and wave reflection in women. Am $\mathbf{J}$ Hypertens. 2005; 18:930-934. [PubMed: 16053989]

Croymans DM, Krell SL, Oh CS, Katiraie M, Lam CY, Harris RA, Roberts CK. Effects of resistance training on central blood pressure in obese young men. J Hum Hypertens. 2014; 28:157-164. [PubMed: 24005959]

Cullen P. Evidence that triglycerides are an independent coronary heart disease risk factor. Am J Cardiol. 2000; 86:943-949. [PubMed: 11053704]

Currie KD, Thomas SG, Goodman JM. Effects of short-term endurance exercise training on vascular function in young males. Eur J Appl Physiol. 2009; 107:211-218. [PubMed: 19554346] 
DeVallance E, Fournier SB, Donley DA, Bonner DE, Lee K, Frisbee JC, Chantler PD. Is obesity predictive of cardiovascular dysfunction independent of cardiovascular risk factors? Int J Obes. $2015 ; 39: 244-253$.

Donley DA, et al. Aerobic exercise training reduces arterial stiffness in metabolic syndrome. J Appl Physiol. 2014; 116:1396-1404. [PubMed: 24744384]

Emre A, Oz D, Yesilcimen K, Sayar N, Ergun D. Impact of the metabolic syndrome on aortic pulse pressure and ascending aortic pulsatility in patients with angiographically normal coronary arteries. Can J Cardiol. 2009; 25:411-414. [PubMed: 19584971]

Fleenor BS, Marshall KD, Durrant JR, Lesniewski LA, Seals DR. Arterial stiffening with ageing is associated with transforming growth factor- $\beta 1$-related changes in adventitial collagen: reversal by aerobic exercise. J Physiol. 2010; 588:3971-3982. [PubMed: 20807791]

Fournier SB, et al. Exercise reveals impairments in left ventricular systolic function in patients with metabolic syndrome. Exp Physiol. 2014; 99:149-163. [PubMed: 24036595]

Fournier SB, Donley DA, Bonner DE, Devallance E, Olfert IM, Chantler PD. Improved arterialventricular coupling in metabolic syndrome after exercise training: a pilot study. Med Sci Sports Exerc. 2015; 47:2-11. [PubMed: 24870568]

Heffernan KS, Fahs CA, Iwamoto GA, Jae SY, Wilund KR, Woods JA, Fernhall B. Resistance exercise training reduces central blood pressure and improves microvascular function in African American and white men. Atherosclerosis. 2009; 207:220-226. [PubMed: 19410255]

Ho SS, Radavelli-Bagatini S, Dhaliwal SS, Hills AP, Pal S. Resistance, aerobic, and combination training on vascular function in overweight and obese adults. J Clin Hypertens. 2012; 14:848-854.

Huang PL. A comprehensive definition for metabolic syndrome. Dis Model Mech. 2009; 2:231-237. [PubMed: 19407331]

Hunter GR, McCarthy JP, Bamman MM. Effects of resistance training on older adults. Sports Med. 2004; 34:329-348. [PubMed: 15107011]

Ibanez J, et al. Twice-weekly progressive resistance training decreases abdominal fat and improves insulin sensitivity in older men with type 2 diabetes. Diabetes Care. 2005; 28:662-667. [PubMed: 15735205]

Jacobson TA, Miller M, Schaefer EJ. Hypertriglyceridemia and cardiovascular risk reduction. Clin Thera. 2007; 29:763-777.

Kato H, Suzuki H, Tajima S, Ogata Y, Tominaga T, Sato A, Saruta T. Angiotensin II stimulates collagen synthesis in cultured vascular smooth muscle cells. J Hypertens. 1991; 9:17-22. [PubMed: 1848253]

Kingwell BA, Arnold PJ, Jennings GL, Dart AM. Spontaneous running increases aortic compliance in Wistar-Kyoto rats. Cardiovasc Res. 1997; 35:132-137. [PubMed: 9302357]

Kraemer WJ, et al. American College of Sports Medicine position stand. Progression models in resistance training for healthy adults. Med Sci Sports Exerc. 2002; 34:364-380. [PubMed: 11828249]

Lakatta EG, Levy D. Arterial and cardiac aging: major shareholders in cardiovascular disease enterprises: part I: aging arteries: a "set up" for vascular disease. Circulation. 2003; 107:139-146. [PubMed: 12515756]

Levy BI, Michel JB, Salzmann JL, Poitevin P, Devissaguet M, Scalbert E, Safar ME. Long-term effects of angiotensin-converting enzyme inhibition on the arterial wall of adult spontaneously hypertensive rats. Am J Cardiol. 1993; 71:8E-16E. [PubMed: 8420241]

Matsuda M, Nosaka T, Sato M, Ohshima N. Effects of physical exercise on the elasticity and elastic components of the rat aorta. Eur J Appl Physiol. 1993; 66:122-126.

Miller MS, Bedrin NG, Ades PA, Palmer BM, Toth MJ. Molecular determinants of force production in human skeletal muscle fibers: effects of myosin isoform expression and cross-sectional area. Am J Physiol Cell Physiol. 2015; 308:C473-C484. [PubMed: 25567808]

Miyachi M, Donato AJ, Yamamoto K, Takahashi K, Gates PE, Moreau KL, Tanaka H. Greater agerelated reductions in central arterial compliance in resistance-trained men. Hypertension. 2003; 41:130-135. [PubMed: 12511542]

Miyachi M, et al. Unfavorable effects of resistance training on central arterial compliance: a randomized intervention study. Circulation. 2004; 110:2858-2863. [PubMed: 15492301] 
Montero D, Roberts CK, Vinet A. Effect of aerobic exercise training on arterial stiffness in obese populations: a systematic review and meta-analysis. Sports Med. 2014; 44:833-843. [PubMed: 24585152]

Rakobowchuk M, McGowan CL, de Groot PC, Bruinsma D, Hartman JW, Phillips SM, MacDonald MJ. Effect of whole body resistance training on arterial compliance in young men. Exp Physiol. 2005; 90:645-651. [PubMed: 15849230]

Rattazzi M, Bertacco E, Puato M, Faggin E, Pauletto P. Hypertension and vascular calcification: a vicious cycle? J Hypertens. 2012; 30:1885-1893. [PubMed: 22871891]

Scuteri A, Najjar SS, Muller DC, Andres R, Hougaku H, Metter EJ, Lakatta EG. Metabolic syndrome amplifies the age-associated increases in vascular thickness and stiffness. J Am Coll Cardiol. 2004; 43:1388-1395. [PubMed: 15093872]

Seals DR. Edward F. Adolph distinguished lecture: the remarkable anti-aging effects of aerobic exercise on systemic arteries. J Appl Physiol. 2014; 117:425-439. [PubMed: 24855137]

Sharman JE, et al. Validation of a generalized transfer function to noninvasively derive central blood pressure during exercise. Hypertension. 2006; 47:1203-1208. [PubMed: 16651459]

Skinner JS, et al. Age, sex, race, initial fitness, and response to training: the HERITAGE Family Study. J Appl Physiol. 2001; 90:1770-1776. [PubMed: 11299267]

Stepp DW. Impact of obesity and insulin resistance on vasomotor tone: nitric oxide and beyond. Clin Exp Pharmacol Physiol. 2006; 33:407-414. [PubMed: 16700872]

Stuart CA, et al. Slow-twitch fiber proportion in skeletal muscle correlates with insulin responsiveness. J Clin Endocrinol Metab. 2013; 98:2027-2036. [PubMed: 23515448]

Tanaka H, Dinenno FA, Monahan KD, Clevenger CM, DeSouza CA, Seals DR. Aging, habitual exercise, and dynamic arterial compliance. Circulation. 2000; 102:1270-1275. [PubMed: 10982542]

Thomas SG, Cunningham DA, Rechnitzer PA, Donner AP, Howard JH. Determinants of the training response in elderly men. Med Sci Sports Exerc. 1985; 17:667-672. [PubMed: 3908879]

Vlachopoulos C, Aznaouridis K, Stefanadis C. Prediction of cardiovascular events and all-cause mortality with arterial stiffness: a systematic review and meta-analysis. J Am Coll Cardiol. 2010; 55:1318-1327. [PubMed: 20338492]

Vyssoulis GP, et al. Differential impact of metabolic syndrome on arterial stiffness and wave reflections: focus on distinct definitions. Int J Cardiol. 2010; 138:119-125. [PubMed: 18804878]

Wilkinson IB, Franklin SS, Cockcroft JR. Nitric oxide and the regulation of large artery stiffness: from physiology to pharmacology. Hypertension. 2004; 44:112-116. [PubMed: 15262901]

Williams MA, et al. Resistance exercise in individuals with and without cardiovascular disease: 2007 update: a scientific statement from the American Heart Association Council on Clinical Cardiology and Council on Nutrition. Phys Act Metab Circ. 2007; 116:572-584.

Yarasheski KE, Pak-Loduca J, Hasten DL, Obert KA, Brown MB, Sinacore DR. Resistance exercise training increases mixed muscle protein synthesis rate in frail women and men $\geq 76 \mathrm{yr}$ old. Am J Physiol. 1999; 277:E118-E125. [PubMed: 10409135] 


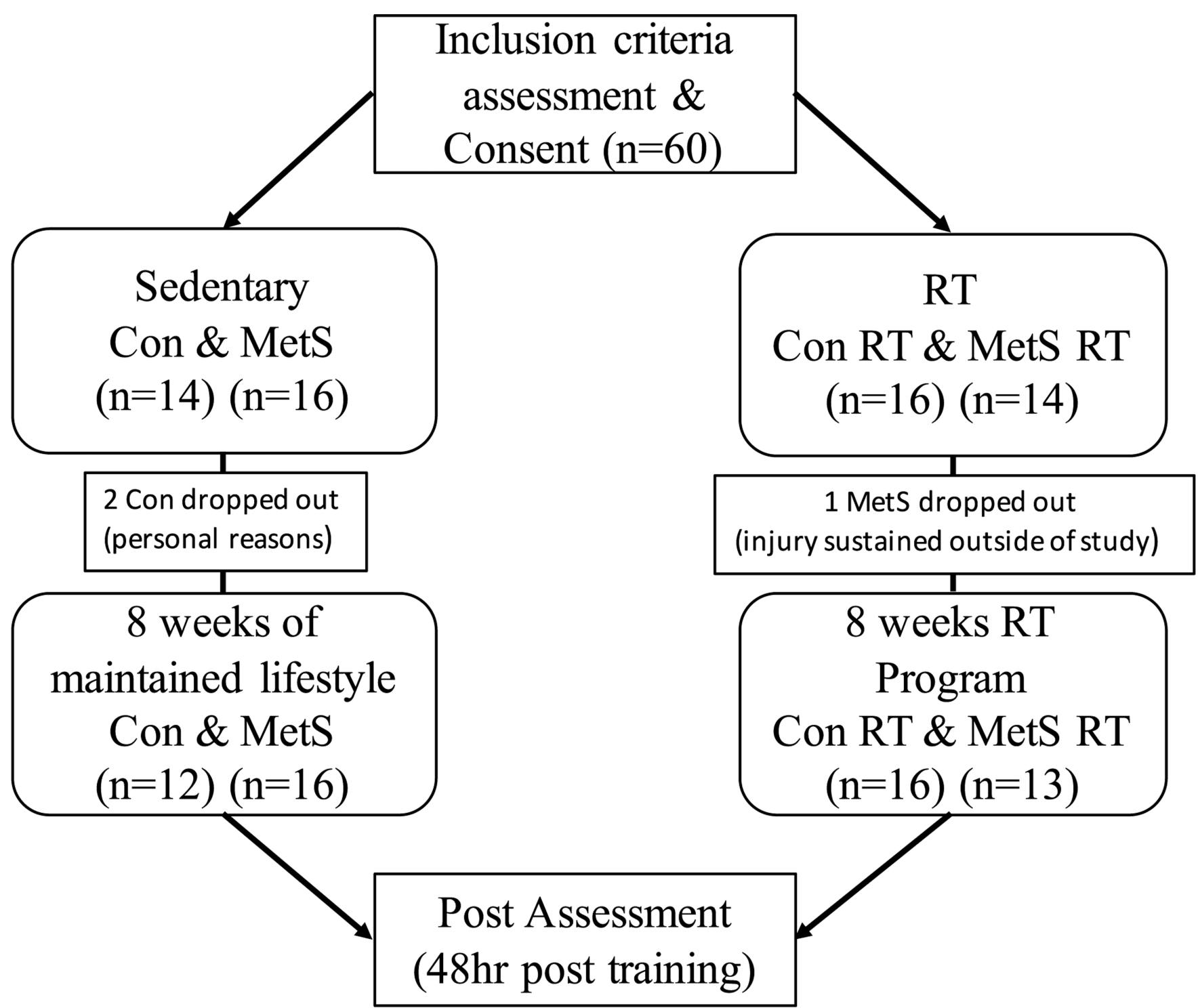

Fig. 1.

Flowchart of study and subjects 


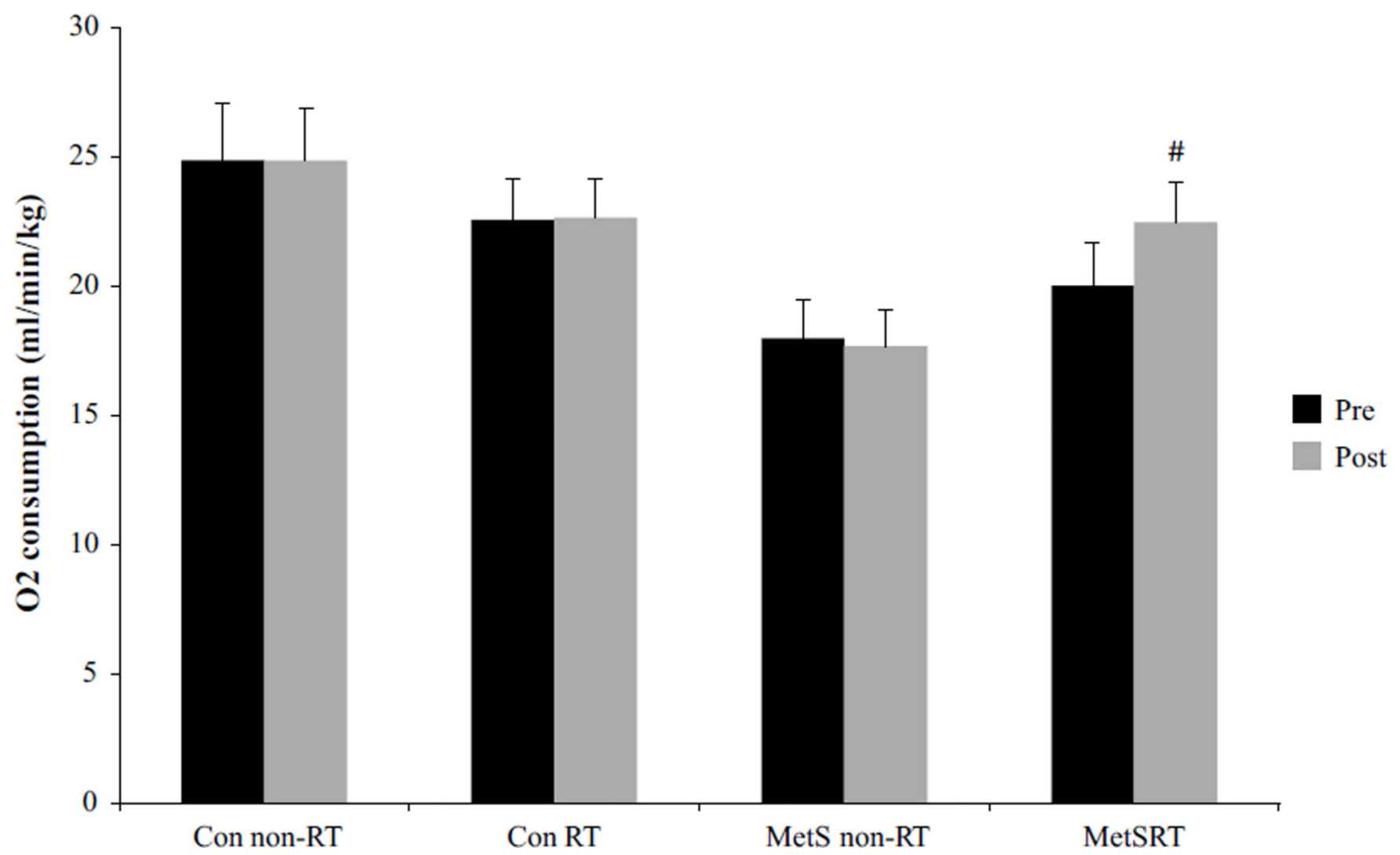

Fig. 2.

Peak aerobic capacity $\left(\mathrm{VO}_{2 \text { peak }}\right)$ for all groups, both pre- and post- 8 weeks of resistance training. Black bars represent pre values, while gray denotes post values. Hash symbol represents a group-by-time interaction for $\mathrm{VO}_{2 \text { peak }}$ in the MetS RT group following training $p \leq 0.05$ 


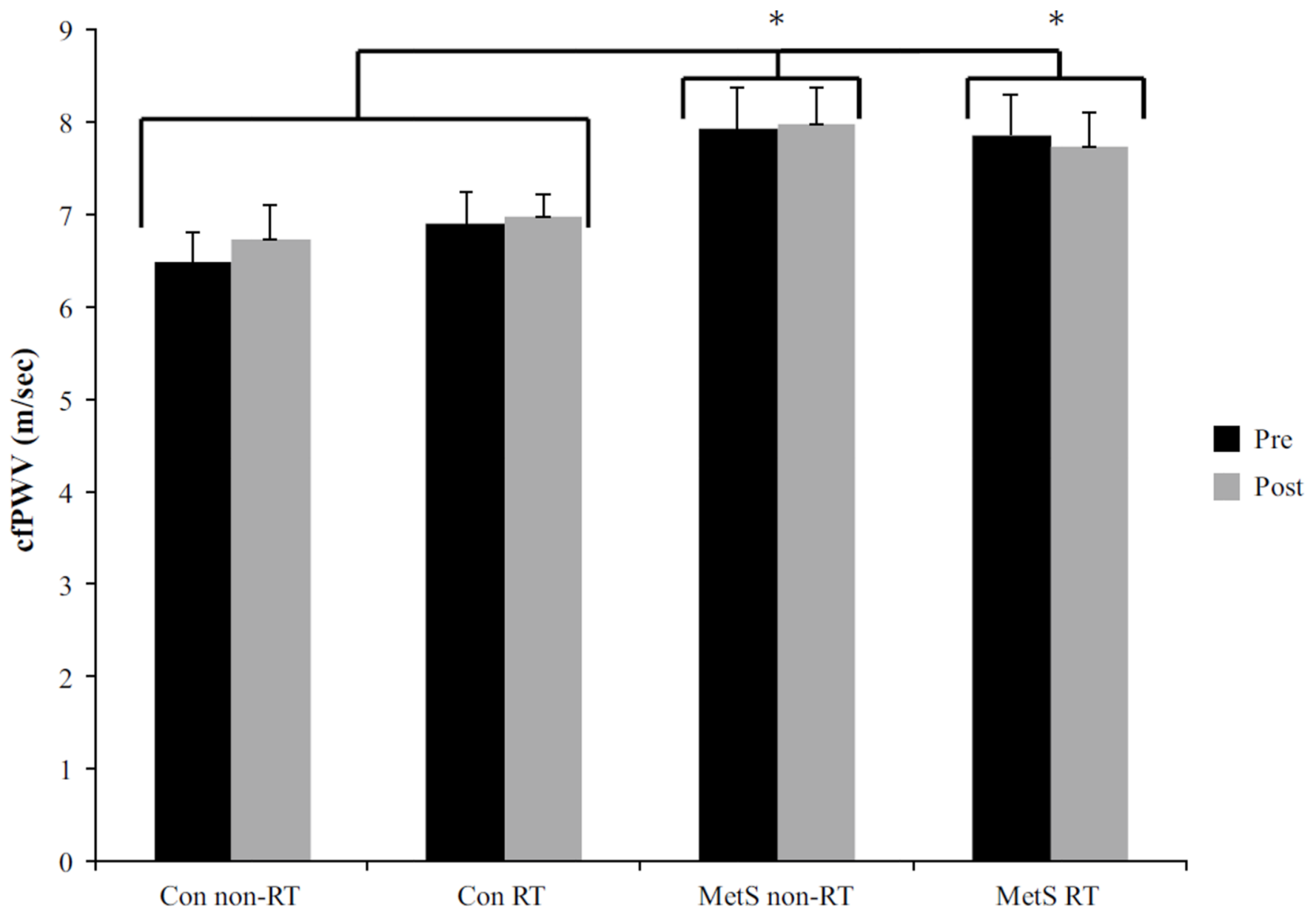

Fig. 3.

Central pulse wave velocity for all groups, both pre-and post- 8 weeks of resistance training. Black bars represent pre values, while gray denotes post values. There was no significant difference in the change from pre to post, but asterisk represents a group difference between MetS vs. Con $p \leq 0.05$ 

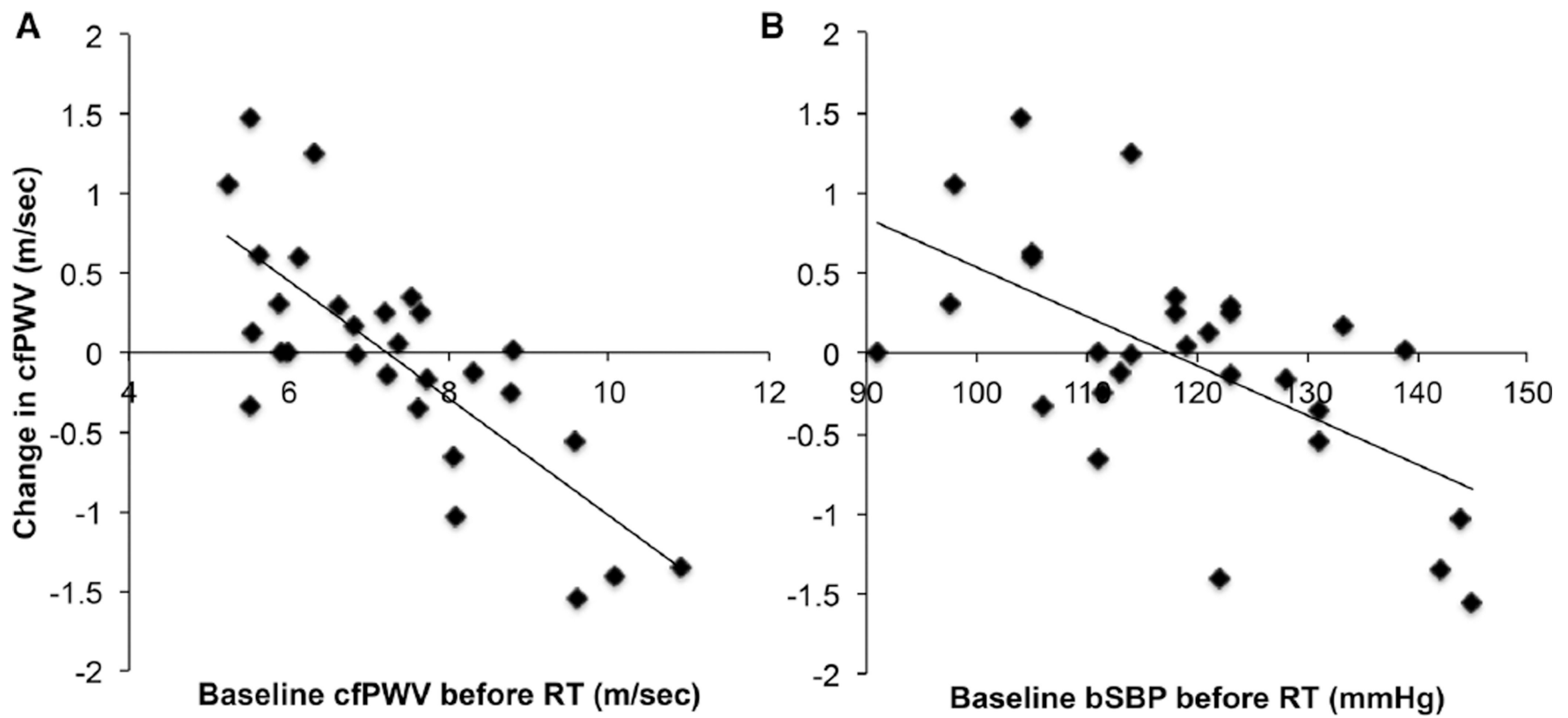

Fig. 4.

Relationship between baseline cfPWV (a, $r=-0.67)$ and bSBP $(\mathbf{b}, r=-0.48)$ to the change in cfPWV elicited by RT 


\section{Table 1}

Baseline clinical characteristics of the study cohort

\begin{tabular}{|c|c|c|c|c|c|}
\hline & \multicolumn{2}{|l|}{ Con } & \multicolumn{2}{|l|}{ MetS } & \\
\hline & $\begin{array}{l}\text { Non-RT } \\
(n=12)\end{array}$ & $\begin{array}{l}\text { RT } \\
(n=16)\end{array}$ & $\begin{array}{l}\text { Non-RT } \\
(n=16)\end{array}$ & $\begin{array}{l}\text { RT } \\
(n=13)\end{array}$ & \\
\hline Age (years) & $44 \pm 3$ & $49 \pm 3$ & $51 \pm 4$ & $51 \pm 3$ & \\
\hline Height $(\mathrm{cm})$ & $166 \pm 2$ & $166 \pm 2$ & $168 \pm 2$ & $166 \pm 2$ & \\
\hline Weight (kg) & $68 \pm 4$ & $67 \pm 3$ & $95 \pm 6^{*}$ & $87 \pm 4^{*}$ & \\
\hline Body fat (\%) & $23 \pm 3$ & $26 \pm 2$ & $38 \pm 2^{*}$ & $38 \pm 2^{*}$ & \\
\hline BMI $\left(\mathrm{kg} / \mathrm{m}^{2}\right)$ & $24 \pm 1$ & $24 \pm 0.9$ & $33 \pm 2^{*}$ & $32 \pm 1^{*}$ & \\
\hline Sex ( $\%$ female $)$ & 75 & 75 & 73 & 69 & \\
\hline Waist $(\mathrm{cm})$ & $82 \pm 4$ & $82 \pm 2$ & $105 \pm 3^{*}$ & $97 \pm 3^{*}$ & \\
\hline Hip (cm) & $100 \pm 3$ & $100 \pm 1$ & $117 \pm 3^{*}$ & $112 \pm 2^{*}$ & \\
\hline Triglycerides (mmol/l) & $1.0 \pm 0.1$ & $0.6 \pm 0.1$ & $1.7 \pm 0.2^{*}$ & $1.7 \pm 0.4^{*}$ & \\
\hline $\mathrm{HDL}(\mathrm{mmol} / \mathrm{l})$ & $1.4 \pm 0.1$ & $1.7 \pm 0.3$ & $1.1 \pm 0.1^{*}$ & $1.2 \pm 0.1^{*}$ & \\
\hline Glucose (mmol/l) & $5.3 \pm 0.2$ & $5.1 \pm 0.2$ & $5.5 \pm 0.1^{*}$ & $5.6 \pm 0.2^{*}$ & \\
\hline HbA1c $(\%)$ & $5.3 \pm 0.1$ & $5.1 \pm 0.1$ & $5.7 \pm 0.1^{*}$ & $5.2 \pm 0.1^{\#}$ & \\
\hline Cholesterol (mmol/l) & $5.1 \pm 0.3$ & $4.6 \pm 0.2$ & $4.7 \pm 0.3$ & $5.1 \pm 0.3$ & \\
\hline Hypertensive (\%) & 0 & 0 & 44 & 46 & \\
\hline Diabetics (\%) & 0 & 0 & 0 & 0 & \\
\hline \multicolumn{6}{|l|}{ Medications } \\
\hline ACE inhibitors (\%) & 0 & 0 & 13 & 0 & \\
\hline $\mathrm{ARB}(\%)$ & 0 & 0 & 13 & 8 & \\
\hline Beta-blockers (\%) & 0 & 0 & 6 & 15 & \\
\hline $\begin{array}{l}\text { Calcium channel } \\
\text { blockers }(\%)\end{array}$ & 0 & 0 & 6 & 0 & \\
\hline Diuertics (\%) & 0 & 0 & 13 & 15 & \\
\hline Anti-platelet (\%) & 0 & 0 & 0 & 0 & \\
\hline Statin $(\%)$ & 0 & 0 & 19 & 23 & \\
\hline \multicolumn{6}{|c|}{ Values are mean \pm SEM } \\
\hline \multicolumn{6}{|c|}{$\begin{array}{l}B M I \text { body mass index, } H D L \text { high-density lipoprotein, } H b A 1 c \text { hemoglobin } \mathrm{A} 1 \mathrm{c}, A C E \text { angiotensin-converting enzyme, } A R B \text { angiotensin II receptor } \\
\text { blocker }\end{array}$} \\
\hline
\end{tabular}




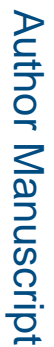

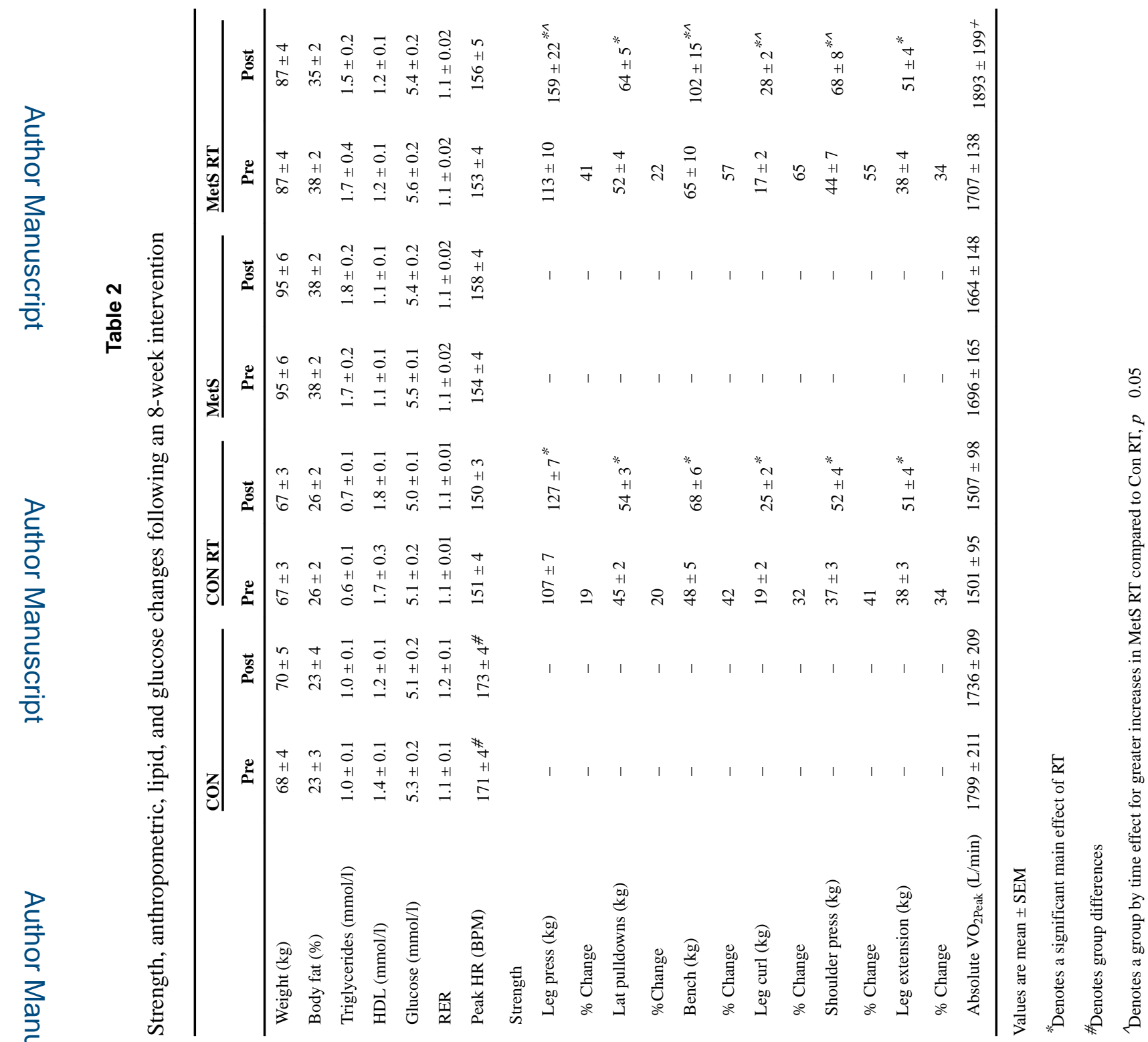

Eur J Appl Physiol. Author manuscript; available in PMC 2017 May 01. 
Table 3

Arterial health parameters pre- and post-hypertrophic resistance training

\begin{tabular}{|c|c|c|c|c|}
\hline & \multicolumn{2}{|l|}{ Con } & \multicolumn{2}{|l|}{$\underline{\text { MetS }}$} \\
\hline & $\begin{array}{l}\text { Non-RT } \\
(n=12)\end{array}$ & $\begin{array}{l}\text { RT } \\
(n=16)\end{array}$ & $\begin{array}{l}\text { Non-RT } \\
(n=16)\end{array}$ & $\begin{array}{l}\text { RT } \\
(n=13)\end{array}$ \\
\hline \multicolumn{5}{|c|}{ IMT (cm) } \\
\hline Pre & $0.63 \pm 0.04$ & $0.62 \pm 0.02$ & $0.71 \pm 0.05^{*}$ & $0.68 \pm 0.02^{*}$ \\
\hline Post & $0.64 \pm 0.04$ & $0.61 \pm 0.02$ & $0.69 \pm 0.03$ & $0.66 \pm 0.03$ \\
\hline \multicolumn{5}{|c|}{ CCA diameter $(\mathrm{cm})$} \\
\hline Pre & $0.58 \pm 0.02$ & $0.57 \pm 0.01$ & $0.60 \pm 0.01^{*}$ & $0.61 \pm 0.02 *$ \\
\hline Post & $0.59 \pm 0.02$ & $0.57 \pm 0.01$ & $0.59 \pm 0.01$ & $0.61 \pm 0.02$ \\
\hline \multicolumn{5}{|c|}{ bSBP (mmHg) } \\
\hline Pre & $118 \pm 2$ & $111 \pm 3$ & $127 \pm 3^{*}$ & $123 \pm 3^{*}$ \\
\hline Post & $116 \pm 3$ & $110 \pm 2$ & $126 \pm 3^{*}$ & $122 \pm 3^{*}$ \\
\hline \multicolumn{5}{|c|}{ bDBP (mmHg) } \\
\hline Pre & $75 \pm 2$ & $71 \pm 2$ & $76 \pm 4$ & $72 \pm 2$ \\
\hline Post & $73 \pm 2$ & $69 \pm 2$ & $77 \pm 3$ & $70 \pm 2$ \\
\hline \multicolumn{5}{|c|}{ bPP (mmHg) } \\
\hline Pre & $43 \pm 3$ & $40 \pm 2$ & $50 \pm 4^{*}$ & $51 \pm 3^{*}$ \\
\hline Post & $44 \pm 3$ & $41 \pm 2$ & $50 \pm 3^{*}$ & $51 \pm 3 *$ \\
\hline \multicolumn{5}{|c|}{ MAP (mmHg) } \\
\hline Pre & $92 \pm 2$ & $85 \pm 2$ & $95 \pm 3^{*}$ & $92 \pm 3^{*}$ \\
\hline Post & $88 \pm 2$ & $84 \pm 2$ & $95 \pm 3^{*}$ & $89 \pm 2^{*}$ \\
\hline \multicolumn{5}{|c|}{ cSBP (mmHg) } \\
\hline Pre & $109 \pm 3$ & $103 \pm 3$ & $115 \pm 3^{*}$ & $115 \pm 3^{*}$ \\
\hline Post & $106 \pm 3$ & $102 \pm 2$ & $116 \pm 3^{*}$ & $113 \pm 3^{*}$ \\
\hline \multicolumn{5}{|c|}{ cDBP (mmHg) } \\
\hline Pre & $76 \pm 2$ & $72 \pm 2$ & $78 \pm 3$ & $73 \pm 3$ \\
\hline Post & $74 \pm 2$ & $70 \pm 2$ & $78 \pm 3$ & $72 \pm 2$ \\
\hline \multicolumn{5}{|c|}{ cPP (mmHg) } \\
\hline Pre & $33 \pm 2$ & $32 \pm 2$ & $38 \pm 3^{*}$ & $42 \pm 3^{*}$ \\
\hline Post & $32 \pm 2$ & $33 \pm 2$ & $38 \pm 3^{*}$ & $41 \pm 3^{*}$ \\
\hline \multicolumn{5}{|c|}{ P1 height (mmHg) } \\
\hline Pre & $24 \pm 2$ & $22 \pm 1$ & $28 \pm 2^{*}$ & $29 \pm 2^{*}$ \\
\hline Post & $25 \pm 2$ & $23 \pm 1$ & $28 \pm 1^{*}$ & $30 \pm 2^{*}$ \\
\hline \multicolumn{5}{|c|}{$\mathrm{AP}(\mathrm{mmHg})$} \\
\hline Pre & $8.5 \pm 1.5$ & $9.2 \pm 1.5$ & $10.0 \pm 2.0$ & $12.5 \pm 1.6$ \\
\hline Post & $8.0 \pm 1.7$ & $9.9 \pm 1.4$ & $10.1 \pm 1.8$ & $11.7 \pm 1.5$ \\
\hline \multicolumn{5}{|c|}{ AGI (\%) } \\
\hline Pre & $25 \pm 4$ & $27 \pm 3$ & $24 \pm 3$ & $28 \pm 2$ \\
\hline
\end{tabular}

Eur J Appl Physiol. Author manuscript; available in PMC 2017 May 01. 


\begin{tabular}{llllll}
\hline & Con & & & MetS & \\
\cline { 2 - 3 } \cline { 5 - 6 } & $\begin{array}{l}\text { Non-RT } \\
(\boldsymbol{n}=\mathbf{1 2})\end{array}$ & $\begin{array}{l}\text { RT } \\
(\boldsymbol{n}=\mathbf{1 6})\end{array}$ & $\begin{array}{l}\text { Non-RT } \\
(\boldsymbol{n}=\mathbf{1 6})\end{array}$ & $\begin{array}{l}\text { RT } \\
(\boldsymbol{n}=\mathbf{1 3})\end{array}$ \\
\hline Post & $22 \pm 5$ & $29 \pm 3$ & $25 \pm 3$ & $27 \pm 2$ \\
\hline
\end{tabular}

Values are mean \pm SEM; there were no group by time interactions

$I M T$ intima-media thickness, $C C A$ common carotid artery, $b S B P$ brachial systolic blood pressure, $b D B P$ brachial diastolic blood pressure, $b P P$ brachial pulse pressure, $M A P$ mean arterial blood pressure, $C S B P$ central systolic blood pressure, $c D B P$ central diastolic blood pressure, $P 1$ height height of the first pressure peak, $A P$ augmentation pressure, $A G I$ augmentation index

*Denotes $p \leq 0.05$ compared to both Con groups 